\title{
DATA ASSIMILATION OF MODEL PREDICTIONS OF LONG-TIME EVOLUTION OF CS-137 DEPOSITION ON TERRAIN
}

\author{
Radek Hofman ${ }^{1,2}$ and Petr Pecha ${ }^{1}$ \\ ${ }^{1}$ Institute of Information Theory and Automation, Academy of Sciences of the Czech Republic \\ ${ }^{2}$ Faculty of Nuclear Sciences and Physical Engineering, Czech Technical University
}

\begin{abstract}
The paper presents a scheme for estimation of spatio-temporal evolution of groundshine dose from radionuclides deposited on terrain in long-time horizon. Groundshine dose mitigation is modeled via semi-empirical formulas taking into account environmental and decay processes. We are aware of the fact that the model is imperfect and special attention is paid to accounting for model error, which is estimated. Methods of Bayesian filtering are applied to the problem. Because of high dimensionality of the problem a parametrization of model error covariance structure has to be introduced. The aim of this paper is to demonstrate utilization of marginalized particle filter for estimating of covariance-model parameters and distribution of groundshine dose.
\end{abstract}

Index Terms - Data assimilation, marginalized particle filter, covariance parametrization, groundshine dose

\section{INTRODUCTION}

Due to global climate changes and growing price of oil we can observe upcoming renaissance of nuclear energy. Along with this trend, demands of responsible authorities on systems for modeling of eventual consequences of possible nuclear accidents increase [1]. Research team from UTIA participated on customization of the RODOS (Rael-time On-line DecisiOn support System) [2] on the Czech conditions. Upon the experience gained in RODOS Data Assimilation working group, we started development of system HARP (HAzard Realistic Predictions system) [3], which is designed for PC-Windows environment. Emphasis is laid on integration of advanced statistical methods developed in UTIA [4] into the assimilation sub-sytem [5]. One of the scenarios being solved is prediction of spatial and temporal evolution of grounshine dose, which occurs due to radioactive material deposited on the ground. The knowledge how fast will radioactivity vanish due to radioactive decay and environmental processes is crucial in planning of countermeasures.

This paper deals with modeling of groundshine evolution in long-time horizon of months or years [6]. As the problem is complex, the groudshine dose evolution model is idealized approximation of the true physical process. The only connection with physical reality are measurements with errors (sparse both in time and in space). In our work, we attempt to make model predictions more reliable in a way of adjusting them toward measurements incoming from terrain and accounting for model error. This process is called data assimilation [7]. Its principle consists in combining of the

This work is part of the grant project GAČR No. 102/07/1596, which is funded by the Grant Agency of the Czech Republic. This work is also supported by the Czech Technical University. information provided by the model with the measured data. Exploiting information on sources of uncertainty, we are able to produce improved estimate of the true situation on terrain.

The general problem of statistical assimilation methods is adequate representation of model error. It has fundamental influence on assimilation performance. We attempt to estimate model error covariance in each time step upon actual measurements. It is represented by an error covariance matrix. As the total number of its parameters is much higher the number of measurements we can't estimate all of them. Simplified covariance model based on idealized assumptions has to be introduced. Model error is formally partitioned into components representing different sources of uncertainty. This is exploited in parametrization of model error covariance via few parameters, which can be easily estimated. For finding the most plausible values of these parameters the similar approach as proposed in [8] or [9] based on modeled-minus-observed residuals is used. Instead of maximum likelihood estimates, we use marginalized particle filter [10] for estimation of both the model error covariance parameters and groundshine dose distribution.

The organization of the paper is as follows. In Section 2, the assimilation scenario is described. Section 3 introduces grounshine dose mitigation model and Section 4 briefly discusses Bayesian filtering. In Section 5, the model error covariance parametrization is presented and the proposed assimilation procedure is explained. In Section 6, experimental results with simulated measurements are presented and the conclusion is given.

\section{ASSIMILATION SCENARIO}

In case of an accidental aerial release of radioactive pollutants into the living environment, the radioactive plume is depleted during passing over the terrain. This phase is called the plume phase. Due to the deposition processes the plume leaves a radioactive trace on the ground.

After the plume phase (when the radioactive cloud exits the area of interest) post-emergency phase follows. It covers latter stages of accident consequence evolution. Post-emergency phase may extend over a prolonged period of several weeks or many years depending on the source of radiation and local conditions. It ends when environmental radiation levels resume to normal. The main exposure pathways in this phase are groundshine and ingestion. The deposited material cause irradiation and through the root system migrates to the edible parts of crops consumed by people and livestock. Among many radionuclides released during emergency situations we focus only on ${ }^{137} \mathrm{Cs}$ since it is one of the most important nuclides in longtime perspective. Its half-time of decay is long (30 years) and also analysis after the Chernobyl accident had shown that it is one of the most significant nuclides in these types of accidents having detri- 
mental long-term effect on population health.

Our assimilation scenario covers the post-emergency phase. The source of pollution is placed into the centre of polar network. We perform our calculations on subset of this network in successive time steps $t \in\left\{1, \ldots, t_{M A X}\right\}$. Groundshine dose in ordered set of analyzed spatial points forms our state vector $\boldsymbol{x}$. Let $\hat{\boldsymbol{x}}_{0}$ be an initial estimate of groundshine dose and $\boldsymbol{P}_{0}$ its corresponding error covariance matrix. This background field is given by probabilistic version of Atmospheric Dispersion Model (ADM) and constitutes the prior characterization of the problem. It is based on segmented Gaussian plume model and it is part of the HARP system, more in [5]. We assume sparse measurements $\boldsymbol{y}_{t}$ of actual gamma dose-rate are available in each time step. These measurements are assumed to be conditionally independent with known error. Assimilation procedure consists of two iteratively repeated steps: In time update step (6) current state estimate together with its error covariance matrix are propagated forward in time. The model error is estimated and accounted for. Following data update step (7) produces so called analysis - adjusts the model prediction to be in accordance with actual measurements.

\section{THE MODEL}

Groundshine dose evolution is modeled according to semi-empirical formulas from Japan model OSCAAR. This abbreviation stands for Off-Site Consequence Analysis code for Atmospheric Releases in reactor accidents. It has been developed within the research activities on probabilistic safety assessment at the Japan Atomic Research Institute [11].

Let $s$ be a spatial coordinate. Relation between $S D(s)$ - the initial ${ }^{137} \mathrm{Cs}$ deposition $\left[B q \times m^{-2}\right]$ and $D_{g}(t, s)-$ the groundshine dose-rate $\left[S v \times s^{-1}\right]$ at time $t$ is given by

$$
D_{g}(t, s)=S D(s) \times S F(s) \times L \times D F_{g} \times R(t) \times E(t),
$$

where $S F(s)$ is the integrated shielding factor for groundshine at location $s, L$ is geometric factor and $D F_{g}=5.86 \mathrm{E}-16 S v \times s^{-1} / B q \times$ $m^{-2}$ is the integrated dose-rate conversion factor for groundshine. $R(t)$ is a unit-less factor taking into account the radioactive decay of the deposited radionuclude with half-time of decay $T_{y}$ (2).

$$
R(t)=\exp \left(-\frac{t}{T_{y}} \ln 2\right)
$$

Term $E(t)$ is a unit-less factor taking into account the decrease of groundshine due to environmental processes, such as radionuclide migration deeper into the soil, weathering, leaching etc. The experiments had shown that the mitigation of groundshine due to environmental removal mechanisms follows relation given by superposition of two exponentials - fast and slow component (3).

$$
E(t)=d_{f} \exp \left(-\frac{t}{T_{f}} \ln 2\right)+d_{s} \exp \left(-\frac{t}{T_{s}} \ln 2\right)
$$

Non-negative parameters $d_{f}$ and $d_{s}$ are weights of decay effect for fast and slow component, respectively. It must hold true that $d_{f}+$ $d_{s}=1 . T_{f}$ and $T_{s}$ are half-times of fast and slow decay terms for groundshine.

Ground deposition model formulas are semi-empirical, it means that some of equation parameters are determined empirically on basis of measurements and the parameter values depend on the local conditions in the area of model application - soil type etc. The dose conversion factor was calculated by the method of Kocher (1980) in which the exposed individual was assumed to stand on a smooth, infinite plane surface with uniform concentration of source of radioactivity.

Some parameters used in ground exposure model are treated as random variables with a given probability distribution. The parameter distribution types were determined for ${ }^{137} \mathrm{Cs}$ from the Chernobyl disaster and they are listed in [11]. The appropriate data for other radionuclides are not available but it is assumed that the long-term influence of the most of them is not significant. For elements with high half-time of decay and similar fixation rates as ${ }^{137} \mathrm{Cs}$ are assumed the same equations of groundshine mitigation. The uncertainty of model parameters plays role in the whole model error and has to be accounted for.

\section{BAYESIAN FILTERING}

Bayesian approach to filtering is applicable to all linear or nonlinear stochastic systems [4], [12]. Let the stochastic system be defined by discrete-time state-space transition equation (4) and observation equation (5)

$$
\begin{gathered}
\boldsymbol{x}_{t}=f\left(\boldsymbol{x}_{t-1}\right)+\boldsymbol{b}_{t} \\
\boldsymbol{y}_{t}=h\left(\boldsymbol{x}_{t}\right)+\boldsymbol{\epsilon}_{t},
\end{gathered}
$$

where $t$ is time index, $\boldsymbol{x}_{t}$ is unobservable system state vector, $\boldsymbol{b}_{t}$ is the dynamic noise vector, $\boldsymbol{y}_{t}$ is the measurement vector and $\boldsymbol{\epsilon}_{t}$ its noise. Generally non-linear operators $f$ and $h$ constitute the transition from prior state to the current one and relation of the measurements to the current state, respectively.

The goal is to acquire posterior density $p\left(\boldsymbol{x}_{t} \mid \boldsymbol{Y}_{t}\right)$ where $\boldsymbol{Y}_{t}=$ $\left\{\boldsymbol{y}_{1}, \ldots, \boldsymbol{y}_{t}\right\}$ are available measurements. Bayesian estimation procedure consists of two iteratively repeated step. The first step transits the state estimate to the next time step according to the probability density function (PDF) $p\left(\boldsymbol{x}_{t} \mid \boldsymbol{x}_{t-1}\right)$ - time update (6). In the second step, the information provided by actual measurements $\boldsymbol{y}_{t}$ is included into the current estimate given by the PDF $p\left(\boldsymbol{x}_{t} \mid \boldsymbol{Y}_{t-1}\right)-$ data update (7).

$$
\begin{gathered}
p\left(\boldsymbol{x}_{t} \mid \boldsymbol{Y}_{t-1}\right)=\int p\left(\boldsymbol{x}_{t} \mid \boldsymbol{x}_{t-1}\right) p\left(\boldsymbol{x}_{t-1} \mid \boldsymbol{Y}_{t-1}\right) d \boldsymbol{x}_{t-1} \\
p\left(\boldsymbol{x}_{t} \mid \boldsymbol{Y}_{t}\right)=\frac{p\left(\boldsymbol{y}_{t} \mid \boldsymbol{x}_{t}\right) p\left(\boldsymbol{x}_{t} \mid \boldsymbol{Y}_{t-1}\right)}{\int p\left(\boldsymbol{y}_{t} \mid \boldsymbol{x}_{t}\right) p\left(\boldsymbol{x}_{t} \mid \boldsymbol{Y}_{t-1}\right) d \boldsymbol{x}_{t}}
\end{gathered}
$$

PDF $p\left(\boldsymbol{x}_{0}\right)$ is called background field or first guess and its choice is important in terms of estimation performance, this is discussed in detail for example in [7].

\subsection{Kalman filter}

Kalman filter (KF) is simple implementation of the Bayesian filter and is widely employed in many fields. Its usage is limited to the case of linear estimation with the Gaussian noise where $p\left(\boldsymbol{x}_{t} \mid \boldsymbol{x}_{t-1}\right)=N\left(\boldsymbol{M} \boldsymbol{x}_{t}, \boldsymbol{Q}_{t}\right)$ and $p\left(\boldsymbol{y}_{t} \mid \boldsymbol{x}_{t}\right)=N\left(\boldsymbol{H} \boldsymbol{x}_{t}, \boldsymbol{R}_{t}\right)$ where $N(\boldsymbol{\mu}, \boldsymbol{Q})$ is Gaussian PDF with mean $\boldsymbol{\mu}$ and covariance $\boldsymbol{Q} . \boldsymbol{M}$ and $\boldsymbol{H}$ are matrices of linear (linearized) operators $f$ and $h$, respectively. Under thess assumptions $(6,7)$ lead to KF equations for time update and data update steps [13]. The equations perform recursive update of the first two moments of estimated Gaussian distribution $p\left(\boldsymbol{x} \mid \boldsymbol{Y}_{t}\right)=N(\hat{\boldsymbol{x}}, \boldsymbol{P})$ - the mean value $\hat{\boldsymbol{x}}$ and its covariance matrix $P$. 


\subsection{Marginalized particle filter}

Particle filter (PF) is more general implementation of Bayesian filter which is applicable to non-linear and non-Gaussian problems [12]. It is based on recursive estimation of the PDF $p\left(\boldsymbol{x}_{t} \mid \boldsymbol{Y}_{t}\right)$ which is represented as a set of so called particles $\boldsymbol{x}_{t}^{(i)}$ and its associated normalized weights $\tilde{q}_{t}^{(i)}$ as $\left.\left\{\tilde{q}_{t}^{(i)}, x_{t}^{(i)}\right\}\right|_{i=1 \ldots M}$. The posterior PDF $p\left(\boldsymbol{x}_{t} \mid Y_{t}\right)$ can be approximated with their help as

$$
p\left(\boldsymbol{x}_{t} \mid Y_{t}\right) \approx \frac{1}{M} \sum_{i=1}^{M} \delta\left(\boldsymbol{x}_{t}-\boldsymbol{x}_{t}^{(i)}\right),
$$

where $\delta$ is Dirac $\delta$-function, $\boldsymbol{x}_{t}^{(i)}$ are samples from approximated PDF. In case that we are not able to sample from $p\left(\boldsymbol{x}_{t} \mid Y_{t}\right)$ we can draw samples $\boldsymbol{x}_{t}^{(i)}$ from a chosen proposal distribution (importance function) $q\left(\boldsymbol{x}_{t} \mid Y_{t}\right)$ and relate them as follows

$$
p\left(\boldsymbol{x}_{t} \mid Y_{t}\right)=\frac{p\left(\boldsymbol{x}_{t} \mid Y_{t}\right)}{q\left(\boldsymbol{x}_{t} \mid Y_{t}\right)} q\left(\boldsymbol{x}_{t} \mid Y_{t}\right) \approx \frac{p\left(\boldsymbol{x}_{t} \mid Y_{t}\right)}{q\left(\boldsymbol{x}_{t} \mid Y_{t}\right)} \frac{1}{M} \sum_{i=1}^{M} \delta\left(\boldsymbol{x}_{t}-\boldsymbol{x}_{t}^{(i)}\right)
$$

Approximation of $p\left(\boldsymbol{x}_{t} \mid Y_{t}\right)$ can be then rewritten as

$$
p\left(\boldsymbol{x}_{t} \mid Y_{t}\right) \approx \sum_{i=1}^{M} q_{t}^{(i)} \delta\left(\boldsymbol{x}_{t}-\boldsymbol{x}_{t}^{(i)}\right), \quad q_{t}^{(i)} \propto \frac{p\left(\boldsymbol{x}_{t}^{(i)} \mid \boldsymbol{Y}_{t}\right)}{q\left(\boldsymbol{x}_{t}^{(i)} \mid \boldsymbol{Y}_{t}\right)}
$$

Distribution function given by (10) has to be normalized (the weights) via constant $c=\sum_{i=1}^{M} q_{t}^{(i)}$. If we choose the posterior PDF from the previous step as proposal distribution in each step, we can via recursive generating samples and evaluating their normalized weights perform Bayesian filtering.

Disadvantage of this method is that we have to be able to generate random samples from complicated distributions and this is for high dimensional problems computationally prohibitive. When the structure of the model $(4,5)$ allows analytical marginalization over a subset of $\boldsymbol{x}_{t}$ we can reduce the computational burden. Let's consider factorization of the state vector $\boldsymbol{x}_{t}=\left[\begin{array}{ll}\boldsymbol{x}_{t}^{l} & \boldsymbol{x}_{t}^{n}\end{array}\right]^{T}$ where $\boldsymbol{x}_{t}^{l}$ is a subset of analytically tractable states and $\boldsymbol{x}_{t}^{n}$ is the rest. Substitution of the factorization into (8) and application of the chain rule gives

$$
p\left(\boldsymbol{x}_{t}^{l}, \boldsymbol{x}_{t}^{n} \mid \boldsymbol{Y}_{t}\right)=p\left(\boldsymbol{x}_{t}^{l} \mid \boldsymbol{x}_{t}^{n}, \boldsymbol{Y}_{t}\right) p\left(\boldsymbol{x}_{t}^{n} \mid \boldsymbol{Y}_{t}\right),
$$

where $p\left(\boldsymbol{x}_{t}^{l} \mid \boldsymbol{x}_{t}^{n}, \boldsymbol{Y}_{t}\right)$ is analytically tractable and $\boldsymbol{x}_{t}^{n}$ is given by the particle filter. Assuming that $\boldsymbol{x}_{0}^{l} \sim N\left(\hat{\boldsymbol{x}}_{0}, \boldsymbol{P}_{0}\right)$ and to be governed by a linear model implies that $p\left(\boldsymbol{x}_{t}^{l} \mid \boldsymbol{x}_{t}^{n}, Y_{t}\right)$ is conditionally linearGaussian and can be computed via Kalman filter [14]. Substitution of (8) into (11) for $\boldsymbol{x}_{t}^{n}$ leads to

$$
p\left(\boldsymbol{x}_{t} \mid \boldsymbol{Y}_{t}\right) \approx \sum_{i=1}^{M} \tilde{q}_{t}^{(i)} \delta\left(\boldsymbol{x}_{t}^{n}-\boldsymbol{x}_{t}^{n,(i)}\right) N\left(\hat{\boldsymbol{x}}_{t}^{l,(i)}, \boldsymbol{P}_{t}^{(i)}\right)
$$

We estimate joint PDF as a mixture of a parametric distribution of Gaussian type and of a nonparametric one. The estimated PDF is represented by a weighted sum of Gaussians, where each particle has a Gaussian distribution attached to it. This modification of PF is called marginalized particle filter (MPF) and details on its implementation are for example in [10], [14].

\section{ASSIMILATION PROCEDURE BASED ON MPF}

The unavoidable condition for application of advanced assimilation methods is knowledge of model error covariance structure represented in each step by its covariance matrix $\boldsymbol{Q}$. The value of $\boldsymbol{Q}$ should reflect total (unknown) model error, it is contribution to the forecast error due to differences between the model and the true process. In KF [13] forecast error covariance matrix $\boldsymbol{P}$ evolves as

$$
\boldsymbol{P}_{t}=\boldsymbol{M}_{t} \boldsymbol{P}_{t-1} \boldsymbol{M}_{t}^{T}+\boldsymbol{Q}_{t},
$$

where $M$ is matrix of linear (linearized) operator for the state transition from $t-1$ to $t$. It is obvious that if $Q$ is neglected, the predicted forecast error will be underestimated.

We attempt to estimate $Q$ in each assimilation step. As the total number of model error covariance parameters to be estimated is much higher than the number of measurements we can't estimate all of them. Simplified covariance model based on idealized assumptions has to be introduced. The idealized model of $Q$ chosen by us has only three parameters $\boldsymbol{\theta}=\left.(\alpha, \beta, L)\right|_{\alpha, \beta, L \geq 0}$, which can be in each time step estimated upon actual measurements,

$$
\boldsymbol{Q}_{t}=\alpha_{t} \times\left[\boldsymbol{Q}_{t}^{(1)}+\beta_{t} \times \boldsymbol{Q}_{t}^{(2)}\left(L_{t}\right)\right]
$$

The model error is partitioned into two components representing different sources of uncertainty. The partitioning has physical background. $\boldsymbol{Q}^{(1)}$ concerns the uncertainty of forecast model parameters introduced in Section 3. This component is found as a covariance of sample obtained via Monte-Carlo simulation with many different model settings. Component $\boldsymbol{Q}^{(2)}$, scaled with $\beta$, is structured, homogeneous and isotropic error. All other sources of uncertainty are accommodated by introduction of $\boldsymbol{Q}^{(2)}$. This component is generated by means of second order autoregressive function $\rho_{L}(r)$ of length-scale parameter $L$ and Euclidean distance between two spatial locations $r$ in meters [15]

$$
\rho_{L}(r)=\left(1+\frac{r}{L}\right) \exp \left(-\frac{r}{L}\right)
$$

The value of length-scale parameter $L$ controls how fast the correlation between two points decreases with their growing distance. The overall covariance is scaled with $\alpha$. This parametrization allows mutual scaling of unstructured noise component $\boldsymbol{Q}^{(1)}$ given upon numerical simulation and "additional" structured noise given by $\boldsymbol{Q}^{(2)}$.

For finding the most plausible values of $\boldsymbol{\theta}$ similar approach as proposed in [8] or [9] based on modeled-minus-observed residuals is used. Instead of maximum likelihood estimates we use MPF introduced in Section 4.2.

When the measurements are available, we can evaluate residuals $\boldsymbol{v}_{t}=\boldsymbol{y}_{t}-\boldsymbol{H} \hat{\boldsymbol{x}}_{t}$. Covariance of $\boldsymbol{v}$ derived in [8] has the form

$$
E\left[\boldsymbol{v}_{t} \boldsymbol{v}_{t}^{T}\right]=\boldsymbol{H}_{t} \boldsymbol{P}_{t} \boldsymbol{H}_{t}^{T}+\boldsymbol{R}_{t}=\boldsymbol{S}_{t}
$$

We assume $\boldsymbol{v}_{\boldsymbol{t}} \sim N\left(\mathbf{0}, \boldsymbol{S}_{t}\right)$. If we substitute (13) into (16) for $\boldsymbol{P}_{t}$ and use covariance parametrization (14) of $\boldsymbol{Q}_{t}$ we obtain

$$
\boldsymbol{S}_{t}(\boldsymbol{\theta})=\boldsymbol{H}_{t}\left[\boldsymbol{M}_{t} \boldsymbol{P}_{t-1} \boldsymbol{M}_{t}^{T}+\boldsymbol{Q}_{t}(\boldsymbol{\theta})\right] \boldsymbol{H}_{t}+\boldsymbol{R}_{t}
$$

Parametrization of model error covariance leads to parametrization of forecast error covariance $\boldsymbol{P}$. The most plausible value of parameters are found in each time step via PF from likelihood $p\left(\boldsymbol{v}_{t} \mid \boldsymbol{\theta}_{t}\right)=$ $N(\mathbf{0}, \boldsymbol{S}(\boldsymbol{\theta}))$ for random parameter vectors $\boldsymbol{\theta}_{t}^{(1)}, \ldots, \boldsymbol{\theta}_{t}^{(M)}$ and actual residual vector $\boldsymbol{v}_{t}$. These parameters are then used in $(13,14)$ for forecast error propagation. Incorporation of this algorithm into $\mathrm{KF}$ assimilation scheme results in MPF for estimation of joint PDF $p\left(\boldsymbol{x}_{t}, \boldsymbol{\theta}_{t} \mid \boldsymbol{Y}_{t}\right)$ which is the mixture of Gaussian and nonparametric distributions.

$$
\underbrace{p\left(\boldsymbol{x}_{t}, \boldsymbol{\theta}_{t} \mid \boldsymbol{Y}_{t}\right)}_{M P F}=\underbrace{p\left(\boldsymbol{x}_{t} \mid \boldsymbol{\theta}_{t}, \boldsymbol{Y}_{t}\right)}_{K F} \underbrace{p\left(\boldsymbol{\theta}_{t} \mid \boldsymbol{Y}_{t}\right)}_{P F}
$$




\section{EXPERIMENTAL RESULTS AND CONCLUSION}

For our research experiments artificial scenario with local rain during the fifth hour of the plume phase was chosen. The rain increases depletion of the plume due the wet deposition. The area of interest is subset of polar network comprising of $N=91$ analyzed points. The measurements were simulated from the measurement equation (5) with linear forward observation operator $\boldsymbol{H}$ where the true initial deposition $\boldsymbol{x}_{0}$ was chosen to be two times higher than the prior estimate $\hat{\boldsymbol{x}}_{0}$. Initial forecast error covariance was set as

$$
\boldsymbol{P}_{0}=2 \overline{\boldsymbol{P}}_{0} \circ \boldsymbol{\Omega}
$$

where $\Omega$ is covariance matrix generated from (15) and the $\circ$ stands for element-wise matrix product (Schur product) [9]. Matrix $\overline{\boldsymbol{P}}_{0}$ was modeled as sample covariance from multiple calls of ADM where the rain intensity was treated as a random variable. Uncertainty of the precipitation intensity is incorporated in $\boldsymbol{P}_{0}$. The element-by-element product with $\Omega$ reduces the strong covariances between distant points.

Initialization of particles in the very first step was following: $\alpha \sim \operatorname{Gamma}(1,1), \beta \sim N\left(10^{2}, 10^{4}\right)$ and $L \sim N\left(10^{3}, 10^{6}\right)$. The prediction was evaluated for six months ahead after the postemergency phase beginning. Measurements were assumed to be available each month, so we performed six successive prediction steps. We also assumed that the measurements were available at time $t=0$ and data update step for improving of prior estimate was performed. At each time step we simulated 10 irregularly spaced measurements. Multinomial resampling described in [16] was used as a resampling algorithm in MPF and particles were resampled each step. Measurement error was set according to expert judgment based on the fact that the smaller measured values have higher relative error than higher values due to the measurement methodology. In each step, first two moments of groundshine dose distribution approximating the truth were predicted and updated .

In the Figure 1, we can see assimilation results in time steps 0,2 , 4 and 6 . The results are in compliance with our expectations for this special scenario - the dose is mitigated and model predictions were successfully adjusted in accordance with the measurements. More general conclusion could not be done until additional tests with various settings are performed. Applicability of the method on higher dimension is still open. Only after cautious and wide verifications the method can be included into the assimilation sub-system.

More comments on experimental results will be given in oral presentation.

\section{REFERENCES}

[1] C. Rojas-Palma et. al, "Data assimilation in the decision support system RODOS," Rad. Prot. Dosim, vol. 104, 2003.

[2] C. Rojas-Palma, "Data assimilation for off site nuclear emergency management," Tech. Rep., SCK-CEN, DAONEM final report, RODOS(RA5)-RE(04)-01, 2005.

[3] P. Pecha, R. Hofman, and P. Kuča, "Assimilation techniques in consequence assessment of accidental radioactivity releases," 2008, ECORAD 2008, Bergen, Norway.

[4] M. Karny et al., Optimized Bayesian Dynamic Advising: Theory and Algorithms, Springer, London, 2005.

[5] P. Pecha and R. Hofman, "Integration of data assimilation subsystem into environmental model of harmful substances propagation," in Harmo11 - 11th Internal Conf. Cambridge, 2007.

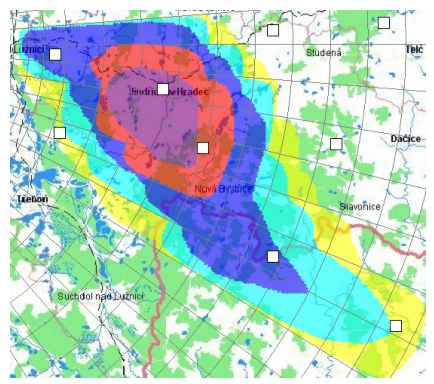

(a) $t=t_{0}$

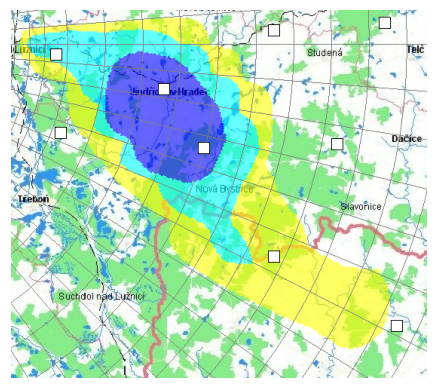

(c) $t=4$ months

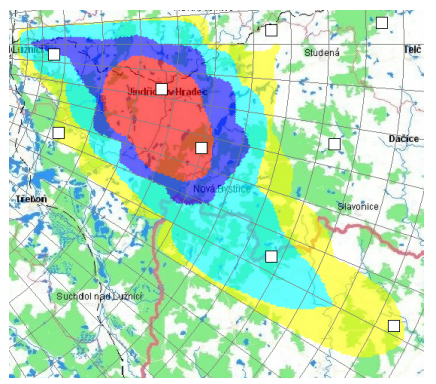

(b) $t=2$ months

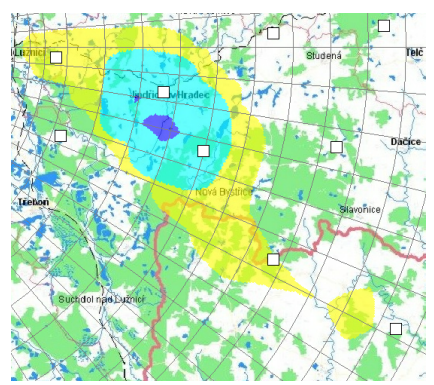

(d) $t=6$ months
Fig. 1. Visualization of assimilated predictions after selected time steps. Measurement locations are indicated with white squares.

[6] F. Gering, W. Weiss, E. Wirth, R. Stapel, P. Jacob, H. Mller, and G. Prhl, "Assessment of evaluation of the radiological situation in the late phase of a nuclear accident," Rad. Prot. Dosim, vol. 109, 2004.

[7] E. Kalnay, Atmospheric modeling, data assimilation and predictability, Cambridge Univ. Press, Cambridge, 2003.

[8] D. P. Dee, "On-line estimation of error covariance parameters for atmospheric data assimilation," Monthly Weather Review, vol. 123, 1995.

[9] Herschel L. Mitchell and P. L. Houtekamer, "An adaptive ensemble Kalman filter," AMS Monthly Weathr Review, vol. 128, pp. 416-433, 1999.

[10] T. B. Schön, R. Karlsson, and F. Gustaffson, "The marginalized particle filter - analysis, applications and generalizations," ESAIM: PROCEEDINGS, vol. 19, pp. 53-64, 2007.

[11] T. Homma and T. Matsunaga, OSCAAR Model - Description and Evaluation of Model Performance, 2006.

[12] A. J. Haug, "A tutorial on Bayesian estimation and tracking techniques applicable to nonlinear and non-Gaussian processes," Tech. Rep., MITRE Corporation, 2005.

[13] R. E. Kalman, "A new approach to linear filtering and prediction problems," Trans. ASME J. Basic Eng., vol. 82, 1960.

[14] V. Šmídl and A. Quinn, "Variational Bayesian filtering," IEEE Transactions on Signal Processing, 2008, to be published.

[15] G. Gaspari and S. E. Cohn, "Construction of correlation functions in two and three dimensions," Quart. J. Roy. Meteor. Soc., vol. 125, pp. 723-757, 1999.

[16] R. Douc, O. Cappé, and E. Moulines, "Comparison of resampling schemes for particle filtering," in Proceedings of the 4th International Symposium. 2005, Image and Signal Processing and Analysis. 